standing of the control of cellular activity has taken a great leap forward. The chemical basis of genetic mutations and their manifestations is clearer. But the manipulation of these to useful ends is not clearly in sight. Already Khorana is forging ahead towards his next goal, the synthesis of a gene. His group has by now almost completed the construction of the polynucleotide sequence corresponding to the gene for alanine transfer R.N.A. in yeast. When complete, this will open up many interesting avenues of research. Not least of these is the possibility of attaching a synthetic gene to a viral or cellular chromosome. The methods are now available and the horizons of genetic engineering, already wide, would be greatly extended thereby.

\section{Haemorrhage and the E.C.G.}

In shock the cardiac output is inadequate to provide normal perfusion of the major organs; the failure is in blood flow rather than blood pressure. The consequent impaired perfusion of tissues leads to hypoxaemia, metabolic acidosis, lactic acidaemia, and hyperkalaemia. The heart and brain are least affected by these changes, since the relatively poor vasomotor innervation of the cerebral and coronary vessels largely excludes them from the generalized vasoconstriction of arterioles and venules in response to the fall in cardiac output. The autonomic nervous system can adequately compensate for $10 \%$ alterations in blood volume, moderate systemic infections, and a good deal of myocardial damage by shunting blood away from visceral organs into cerebral, coronary, and skeletal muscle beds.

The perfusion of the coronary arteries is, however, largely dependent on the pressure in the aorta, and in patients with ischaemic heart disease-in whom the coronary vessels are already maximally dilated-aortic blood pressure becomes an even more important factor in assuring adequate coronary blood flow. As long ago as 1939 C. K. Friedberg and $\mathrm{H}$. Horn ${ }^{1}$ observed myocardial necrosis due to functional coronary insufficiency without coronary occlusion, in both experimental animals and in patients with haemorrhagic shock. If hypotension is severe it is not surprising that ischaemic changes in the electrocardiogram become apparent. As more than $60 \%$ of patients in shock, regardless of its cause, have increased serum transaminase and lactic dehydrogenase activity due to ischaemic injury to the liver, the differentiation of cardiogenic shock from other causes of shock may give rise to difficulty. In a recent article in Diseases of the Chest $\mathrm{D}$. Scherf and C. Bornemann ${ }^{2}$ remind us that patients may present with a picture of tachycardia, pallor and sweating, precordial pain, and abnormalities in the electrocardiogram, all of which are a result of gastrointestinal haemorrhage. This is important, since internal haemorrhage may not be accompanied by haematemesis, and melaena may be delayed for several days. If such a patient is treated with anticoagulants on the basis of a diagnosis of myocardial infarction the result would be disastrous. Scherf and Bornemann report $T$ wave inversion occurring with severe acute haemorrhage 14 times in a series of 15 successive

1 Friedberg, C. K., and Horn, H., f. Amer. med. Ass., 1939, 112, 1675. 2 Scherf, D., and Bornemann, C., Dis. Chest, 1968, 53, 99. instances of patients with acute haemorrhage from peptic ulceration. These changes occur within a few hours after haemorrhage and last for several days. Usually only the $T$ waves are affected, but occasionally the $S-T$ segments may be depressed. When acute haemorrhage induces anginal pain with hypotension, sweating, and abnormal electrocardiographic change a diagnosis of myocardial infarction may mistakenly be made.

\section{Modified Rapture}

Many countries are now reporting standardized death rates lower than those in England and Wales. Despite the doubts that must attend any international comparison the picture may be accepted as true enough to stimulate further efforts here to improve matters. According to the latest report from the Chief Medical Officer of the Ministry of Health ${ }^{1}$ the statistical indices of mortality in Britain could be substantially improved. It is worth noting that general and infant mortality are both worse in Scotland and Northern Ireland than in England and Wales. In general John Bull is in pretty good shape, but the C.M.O.'s report ought to trouble the complacent expression habitual to him.

One satisfactory fall in mortality is to be seen on the roads. The C.M.O. notes a "striking reduction" in the last quarter of 1967 after the introduction of alcohol tests. But if man's irresponsibility in relation to one drug has been somewhat curbed, it has found increasing expression in the misuse of others. Outstanding among these is still tobacco, the main cause of lung cancer. The death rate from this disease again rose among both men and women in 1967. In fact the increase for men was the largest since 1959. All told, some 28,000 persons died of it in the year under review. Other poisons are also proving to be fatally attractive to abuse. The report observes that hospital admissions of cases of selfpoisoning continued to rise, and cites the conclusion of a subcommittee (whose report is to be published) that "the majority of deaths in adults are the result of deliberate selfadministration and that accidental death from ingested poisons is largely restricted to children under 10 years of age." (The circumstances of accidental poisoning in childhood are reviewed elsewhere in this issue of the B.M.F. (p. 245) by R. H. Jackson and his colleagues.) It can cause no surprise, either, that the report's chapter on mental health opens with a section on drug addiction, a still growing menace to health.

The annual death rates from cancer of the breast in women, even when standardized for age, have shown a disquieting tendency to rise. Fluctuations are admittedly fairly small, but 1964 and 1967 provided the highest rates in recent years, and there is certainly no sign of any decrease. The report also draws attention to a question that has been aired before in these columns-namely, whether there is a non-industrial risk of developing lung cancer from asbestos. An industrial risk from asbestos dust is known and guarded against, but so numerous are the uses to which this easily worked and noninflammable material is now being put that dust from it may be more prevalent in the general environment than is realized.

1 On the State of the Public Health: Annual Report of the Chief Medical Officer of the Ministry of Health for the Year 1967. 1968. H.M.S.O. 